\title{
Recurrent Differentiated Thyroid Cancer
}

\author{
Sami Tannouria ${ }^{\mathrm{a}}$ David Goldenberg ${ }^{\mathrm{b}, \mathrm{c}}$
}

\begin{abstract}
Despite high prevalence of malignancy of the thyroid, recurrent thyroid cancer remains a poorly understood entity. This paper aims to consolidate research and literature specifically on recurrent disease by discussing its epidemiology, genetics, surveillance, treatment and outcomes. Mutations in the BRAF and RET genes predispose to recurrent disease. Currently utilized surveillance methods include thyroglobulin monitoring, radioiodine body scanning, PET/ $\mathrm{CT}$, and neck ultrasound. Surgical reoperation is optimal treatment of choice. Long term outcomes still remain poorly described.
\end{abstract}

Keywords: Thyroid; Cancer; Thyroid cancer; Recurrent differentiated thyroid; Thyroid cancer; Recurrent; Differentiated; Ultrasound; PET-CT; Review

\section{Epidemiology}

Thyroid cancer is the most common endocrine malignancy. In 2010 there were 44,670 new cases and 1,690 deaths from all thyroid cancers [1]. Between $5-15 \%$ of all thyroid nodules are discovered to be malignant. Thyroid follicular epithelial cells give rise to follicular carcinomas, hurthle cell carcinoma, papillary carcinoma, and less often, undifferentiated carcinomas such as anaplastic or poorly differentiated carcinoma [2]. Ninety percent of thyroid cancers are of the

Manuscript accepted for publication April 19, 2012

${ }^{a}$ The Pennsylvania State University - Milton S. Hershey Medical Center, College of Medicine, USA

${ }^{b}$ Division of Otolaryngology-Head and Neck Surgery, The Pennsylvania State University, The Milton S. Hershey Medical Center, 500 University Drive, P.O. Box 850 H091, USA

${ }^{\mathrm{c} C}$ Corresponding author: David Goldenberg.

Email: dgoldenberg@hmc.psu.edu

doi: $10.4021 / \mathrm{jcs} 39 \mathrm{w}$ differentiated types, papillary or follicular [3], while less than $5 \%$ are anaplastic. Often, recurrent disease retains identical histological characteristics as the primary malignancy, but also may recur with a less differentiated histology [2]. Literature exists on rates of recurrence but it is limited and dated. Published rates range from a $5-25 \%$ recurrence rate in papillary cancer and $25-61 \%$ for various stages of follicular carcinoma [2, 4-7]. A further confounding issue is that most published literature lumps both persistent and recurrent disease as a single entity despite these being entirely distinct forms of disease.

Risk factors and prognostic criteria for recurrent thyroid cancer differ from those of primary disease. Survival in primary well differentiated thyroid cancer is inversely related to both patient age and tumor size $[8,9]$. Studies have shown that only extrathyroidal extension either via surgical pathology or lymph node involvement at the time of resection is actually a risk factor for developing recurrent disease [10]. One such study ( $\mathrm{N}=246)$, higher pTNM staging, age, and primary tumor size were not significantly associated with recurrence [8]. Primary cases of aggressive or highly invasive histological variants are at an increased risk for recurrence $[2,11]$. Raef et al. followed 100 consecutively diagnosed patients with differentiated (papillary $76 \%$, follicular variant $14 \%$, hurthle $4 \%$, tall cell and sclerosing $2 \%$, pure follicular $4 \%)$ thyroid cancer. Nine of these cases recurred within the year after remission was achieved. Fifty patients had either recurrent or persistent disease in this cohort, and they tended to be older, had a higher TNM stage, and higher post surgery thyroglobulin levels as compared to patients who remained in remission. Gender was not determined to be a significant factor for recurrence in this study [12]. While there is no definitive answer on the effect of nodal metastasis on survival, it is generally agreed that nodal involvement increases rates of recurrent disease [13]. Locoregional recurrence in papillary thyroid cancer more often occurs in the lateral neck compartment $(8.2 \%, \mathrm{~N}=342)$ [10].

In a study of multicentric papillary thyroid carcinoma, Lin et al demonstrated higher rates of recurrence $(20.2 \%)$ than that of those patients with unifocal disease $(n=1682)$. Of those with multicentric disease with greater than 5 foci, $45.8 \%$ recurred [14]. 
Tuttle et al devised a recurrence risk stratification scheme for patients with thyroid cancer. Low risk patients are defined as individuals at any age with PTC and primary tumor size of $<1 \mathrm{~cm}$ without lymph node involvement. Intermediate risk patients are between 20 - 60 years of age with PTC with extrathyroid or vascular extension. The primary tumor size of must be between 1 and $4 \mathrm{~cm}$ with or without lymph node involvement. High risk patients are classified as $<20$ or $>60$ years old with variants other than PTC or gross extrathyroid extension or vascular invasion. These patients have a primary tumor size of $>4 \mathrm{~cm}$ and are positive for lymph node involvement [15].

\section{Mutations and Recurrent Thyroid Cancer}

BRAF mutations and RET/PTC rearrangements are often found in papillary thyroid carcinomas. Rarely, BRAF mutations can initially be absent in primary disease but then occur in the process of PTC recurrence [16]. Recurrence is more likely in the presence of a BRAF mutation and some authors suggest total thyroidectomy if FNA genetic analysis are positive for such [17-21]. BRAF mutated PTC was associated with at $23 \%$ recurrence rate in one study as compared to $9 \%$ in PTC without BRAF mutation [22]. In another examination of 54 cases of recurrent PTC, BRAF mutations were detected in $77.8 \%$ of cases. Only $16.7 \%$ of these cases exhibited RET/PTC rearrangement. Both mutations were simultaneously present in $9.3 \%$ of patients with recurrent disease, a much higher percentage than the prevalence of dual mutations in primary disease. These findings support the idea that BRAF mutations, as well as dual mutations, are significantly associated with recurrent papillary thyroid carcinoma [23]. A BRAF V600E mutation in patients older than 65 with papillary thyroid cancer has been associated with a higher incidence of recurrence than in younger patients $(10 \%$ and $2 \%$, respectively). This mutation itself also predisposed to recurrence in $13 \%$ of cases as compared to $2 \%$ of BRAF negative patients [24]. BRAF mutations may also be associated with recurrent disease lacking radioiodine avidity [16]. Cradic et al developed a PCR-based blood test to detect BRAF mutations. This technique may be useful for detecting recurrence of PTC although this has not yet been validated [18].

Both VEGF and VEGF-C (a lymphangiogenic factor) are elevated in cases of recurrent papillary thyroid carcinoma and, like in other cancers, are proposed to play a role here in growth, metastasis and recurrence [25]. Papillary thyroid carcinomas with recurrence appearing in the contralateral lobe expressed identical genetic mutations in both the primary and recurring tumor. This suggests that local metastasis plays an important role in recurrent disease [26].

Far less is described about the role of genetics in recurrent follicular thyroid cancer. Primary disease is usually as- sociated with a mutation in either RAS or in PAX8/PPAR $\gamma$ $[20,21]$.

\section{Surveillance and Diagnosis}

The 2009 ATA Guidelines define absence of persistent tumor as an undetectable serum $\mathrm{Tg}$ and negative imaging studies post-thyroidectomy or near-total thyroidectomy with remnant ablation [3]. One study showed that cases of multicentric papillary thyroid carcinoma that exhibited disease recurrence were diagnosed within one year post thyroidectomy $52.9 \%$ of the time [14]. Recurrent thyroid cancer typically appears with metastatic disease in the cervical lymph nodes $(85 \%)$, with $32 \%$ having thyroid bed recurrence and $12 \%$ having distant metastasis [27].

The two methods to detect recurrence or metastasis are by measurement of serum thyroglobulin (Tg) or by I-131 whole body scans (WBS). Certain patients test negative for serum Tg but are I-131 WBS positive despite the higher sensitivity of serum Tg. Patients with this paradoxical finding were more likely to have local disease recurrence or metastasis, and thus it is recommended that patients receive an iodine WBS post-ablation therapy regardless of postoperative serum Tg [28]. Serum Tg may not be an adequate test to predict recurrent disease [9] although a stimulated Tg may be more useful for this purpose [29].

One study examined patients deemed disease free after thyroidectomy and radioiodine ablation and who were taking levothyroxine. They were challenged with recombinant human TSH (rhTSH) and stratified into 3 groups based on their measured thyroglobulin $(\mathrm{Tg})$ response. Rates of cancer recurrence were measured amongst these groups and it was found that those with higher rhTSH stimulated thyroglobulin levels exhibited more recurrence. Challenged Tg values of $2.5 \mathrm{ng} / \mathrm{mL}$ or higher predicted recurrence with sensitivity and specificity of 80 and 97 respectively [30]. Patients who are post-thyroidectomy with ablation may have undetectable stimulated thyroglobulin levels. A marker of recurrent thyroid cancer may be a previously undetectable thyroglobulin level which has become measurable again. Nascimento et al showed that repeating TSH-Tg measurements 6 - 18 months post surgery in patients with undetectable postoperative stimulated $\mathrm{Tg}$ does not yield information on recurrence [11].

The presence of Anti-thyroglobulin antibody (TgAb) further complicates serum $\mathrm{Tg}$ measurements as a screening tool for recurrence. The presence of $\mathrm{TgAb}$ is higher in patients with differentiated thyroid cancer than in the general population and thus may cause falsely lowered measured $\mathrm{Tg}$ concentration. TgAb levels should be measured especially in patients with initially undetectable serum $\mathrm{Tg}$ values post surgery and ablation. Downward trending serial $\mathrm{TgAb}$ measurements are a marker of good prognosis and 
conversely an increasing trend raises the risk of recurrence. Taken together, values of $4.45 \mathrm{ng} / \mathrm{mL}$ and $27.81 \mathrm{U} / \mathrm{mL}$ for $\mathrm{Tg}$ and $\mathrm{TgAb}$ respectively were found to be significantly more sensitive and just as specific for detecting recurrence as single $\mathrm{Tg}$ measurements (Sensitivity increased from $52.5 \%$ to $78.3 \%$, whereas specificity decreased from $91.7 \%$ to $90 \%$ ) [31].

Imaging modalities such as neck ultrasound, CT, MRI, and FDG-PET have all been evaluated as tools in the detection of thyroid cancer recurrence. Neck ultrasound, according to the 2009 American Thyroid Association guidelines, should be used to survey the thyroid bed and cervical lymph node compartments at 6 - 12 months and beyond. Neck US is now a preferred detection modality along with serum $\mathrm{Tg}$ over diagnostic whole body scanning (DxWBS) [3]. Neck US is able to detect both differentiated and undifferentiated (not typically radioiodine avid) thyroid cancers, whereas DxWBS detects only radioiodine avid cancers [32]. DxWBS can be performed after 3 weeks of thyroid hormone therapy withdrawal [33].

Patients with intermediate or high recurrence risk may benefit from DxWBS 6 - 12 months after ablative therapy. Isotopes such as I-123 or I-131 should be used to perform DxWBS. DxWBS is not indicated in patients on thyroid hormone replacement with undetectable serum $\mathrm{Tg}$ and $\mathrm{TgAb}$, and an unrevealing neck ultrasound [3]. Administration of a diagnostic I-131 dose may cause thyroid stunning and a therapeutic follow-up dose of I-131 will not be adequately absorbed for proper imaging. I-123 imaging is of better quality but its greater expense and short half life makes its usage more difficult [32].

Some differentiated thyroid cancers may lose their ability to take up radioactive iodine, but concurrently begin taking up more FDG (2-Deoxy-2(18F)-fluoro-D-glucose). This "flip-flop" phenomenon is taken advantage of by FDG-PET scanning in Tg positive yet WBS and ultrasound negative recurrence [34-39]. Sensitivities of PET and PET-CT in the detection of recurrent PTC were calculated to be $77-85 \%$ and $82-84 \%$ in a meta-analysis [40]. Choi et al calculated the sensitivity and specificity of PET scanning to be 56.6 and $52.2 \%$ in detection of recurrent PTC in a series of 76 cases [35]. PET scanning is not effective as a first line diagnostic for detection of recurrent disease, but there may be a yet to be defined role for concurrent WBS and PET scans [4144].

Ultrasound-guided fine needle aspiration (US-FNA) is a safe and effective tool to diagnose and characterize recurrence of DTC [45]. Examination of the thyroid bed and the internal jugular chain lymph nodes should be conducted, and when suspicious of an aggressive recurrence, examination should extend to the supraclavicular, lateral cervical and submandibular lymph nodes. Inferior pretracheal lymph nodes should be scanned in patients who had isthmic or lower pole cancer [46]. Some research has demonstrated a $27 \%$ recur- rence rate of DTC diagnosed by US-FNA and these recurrences were associated with elevated $\mathrm{Tg}$ levels as well as with ultrasound abnormalities such as irregular margins or non-ovid lesion shape. This modality may be less useful in patients without such characteristics [47]. The drawback of ultrasound is that it inadequately visualizes the paravertebral, retrotracheal and retropharyngeal regions as well as nodal chains close to the skull. Fortunately most recurrence does not present in these areas [27].

Tuttle et Al recommend different postoperative surveillance for each of his risk strata for the first 1 - 2 years post treatment. Low risk patients should have suppressed Tg measured every 6 months and a neck ultrasound yearly for two years. These patients do not need stimulated Tg measured, nor do they need DxWBS [48], MRI/CT or FDG-PET. Intermediate risk patients need a suppressed $\mathrm{Tg}$ measured every 6 months and a stimulated Tg measured $12-24$ months after treatment. They should undergo neck US yearly for 2 years and should receive radioactive iodine (RAI) DxWBS at 12 - 24 months. They do not require MRI/CT, or FDGPET. High risk patients should receive the same surveillance as intermediate risk patients with the addition of cross sectional imaging such as CT/MRI if their Tg measurements are elevated or if there is clinical suspicion. Furthermore, they should receive FDG-PET scanning if a Tg measurement is elevated but their RAI DxWBS is negative [15].

\section{Treatment Modalities}

Surgical therapy is widely regarded as definitive treatment for recurrent thyroid carcinoma. Central compartment dissection for recurrent PTC, while carrying an increased risk for hypoparathyroidism and recurrent laryngeal nerve injury, remains the treatment modality of choice for regional recurrent or metastatic disease [13, 49]. Central neck dissection entails removal of all soft tissues in level VI and VII neck compartments in a patient who previously underwent thyroidectomy or central lymph node dissection. It is recommended to obtain cross sectional imaging such as CT or PET-CT for operative planning because physical examination and ultrasound can miss pathological mediastinal lymphadenopathy [50]. Recurrent laryngeal nerve identification and monitoring as well as retention of parathyroid gland vascular pedicles reduce reoperative morbidity in central compartment dissection [51] although the risk of nerve injury is greater in reoperation than primary surgery. Early visual identification of the nerve is of utmost importance and is the gold standard measure to prevent nerve injury in both primary surgery and reoperation. Preoperative laryngoscopy should be performed to evaluate for RLN injury as this may influence the operative approach [50]. Intraoperative nerve monitoring may be particularly helpful in revision surgery [52]. It is particularly important 
that all fibroadipose tissue posterior to the strap muscles be removed in central compartment reoperation because this tissue contains paratracheal lymph nodes which may harbor cancer [53].

Recurrence in a remnant thyroid is far more often operable than recurrence within the thyroid bed. Reoperation should be done through the prior operative site and efforts to should be undertaken to maintain as much of the anatomy undisrupted, despite the presence of fibrosis and scar tissue from the previous surgery. Identification of the recurrent laryngeal nerve is of utmost importance and may be difficult intraoperatively without the use of nerve monitoring devices due to the possibility that the nerve is encased in fibrotic tissue. Dissection along the carotid artery inferiorly may allow access to undisrupted tissue planes and may assist in locating and identifying the nerve. The dissection should include all remnant lymphatic tissue from the trachea to the carotid artery. The superior and inferior borders of the dissection should extend from the cricoids to the superior edge of the clavicle. Picking out individual lymph nodes which appear diseased is highly discouraged. Due to the fibrosis and scarring, intraoperative ultrasound can be very useful for identifying disease $<20 \mathrm{~mm}$ [54].

Preservation of the inferior thyroid artery is recommended as it reduces parathyroid devascularization. Due to increased scar tissue and fibrosis, liberal frozen section pathology should be utilized to ensure that parathyroid glands are not inadvertently removed [53]. Patients with low intact serum PTH are associated with postoperative hypocalcemia $[55,56]$. Reoperative central lymph node dissections (CLND) were found to have a lower rate of transient postoperative hypocalcemia as compared to initial dissections. Inadvertent removal of parathyroid tissue is common in reoperation but is not associated with postoperative hypoparathyroidism [57]. No comparison has yet been done of the risk of transient or permanent hypoparathyroidism post lateral versus central compartment dissection. The role of prophylatic CLND for unenlarged central compartment nodes remains controversial due to operative risks [4]. Most importantly, reoperation is effective at treating recurrent disease [29].

Recurrence in the lateral neck discovered by US should be further evaluated with FNA to identify regional metastasis. Recurrent disease most often occurs in levels II-V involving the transverse cervical and spinal accessory lymph nodes [58]. Lateral compartment dissection carries its own set of complications, which include thoracic duct injury, internal jugular vein injury, carotid artery damage, spinal accessory nerve damage and brachial plexus injury, among others $[13,59]$. Nononcologic structures such as the jugular vein, spinal accessory nerve, and sternocleidomastoid should be retained unless directly invaded by tumor recurrence. Lymph node resection should include the highest affected node along with its drainage basin inferiorly. Again, selection of individual diseased lymph nodes is discouraged in lateral dissection and proper technique is to remove an entire nodal basin [54].

Central or lateral lymph node dissection (LND) for recurrent PTC may result in cure (defined as total or near total $\mathrm{Tg}$ reduction) in $72 \%$ of cases [60]. There is no difference in the rate of central versus lateral recurrence after a primary or reoperative central lymph node dissection [4]. Critics of the trend towards prophylactic neck dissection put forth that extrathyroidal extension (ETE) and lymphovascular invasion are independent predictors of regional recurrence and may be of little benefit to patients without these findings. In those with gross ETE there may be an additional role for contralateral central compartment dissection as well as dissection of ipsilateral level III and IV [10, 61].

Laryngotracheal involvement can occur in up to $16 \%$ of cases. Adherence to the trachea resulting in a shaving resection or more significant involvement requiring tracheal resection with end to end anastomosis are both appropriate as long as the tumor is completely excised. It is critical implement a resection which offers the lowest perioperative morbidity as well as the best quality of life. More significant laryngotracheal involvement requiring complex reconstruction suffer from increased rates of complications and perioperaive death [54].

Radioguidance is emerging as a tool in revision thyroid surgery that can be particularly useful after normal anatomy has been disrupted or there has been incomplete resection of tissues. As most recurrent disease is not iodine avid, radiopharmaceuticals such as ${ }^{99 \mathrm{~m}} \mathrm{Tc}$ sestamibi or ${ }^{18} \mathrm{~F}-\mathrm{FDG}$ can be used to tag remnant tissues in difficult or previously undetected locations [62].

The use of same-day US along with preoperative imaging in resection of recurrent PTC leads to greater chance of success without impacting mean operating time. Preoperative US-guided injection of blue dye into a focus of recurrent thyroid cancer has also been successfully used to intraoperatively localize disease recurrence [63-65].

Aggressive histological variant cancers should have surgical treatment followed up with radioiodine ablation. Ablation does not need to be implemented in patients postoperatively when their measured Tg is undetectable and they have a normal neck ultrasound [11]. Ablation may be less effective in PET positive lesions as they often have reduced ability to take up radioiodine [66].

External beam radiation can be used on non-radioiodine avid tumors, painful bony metastasis, and tumors in regions that may cause significant anatomic complications but cannot be surgically treated. Recurrence is more often treated with radiation, particularly when there is extracapsular extension from the remnant thyroid [54]. Stereoctactic body radiotherapy [33], radiofrequency ablation, ethanol injection, and chemoembolization have been used in certain cases although as of yet chemotherapeutic treatment of DTC has 
shown little to modest response [67].

\section{Molecular Strategies}

The mainstay of medical treatment of DTC is thyroxine replacement, which negatively feeds back on TSH thus inhibiting TSH-dependent tumor growth. Poorly differentiated cancers may not be as responsive to this modality [33].

Both VEGF and VEGF-C (a lymphangiogenic factor) are elevated in cases of recurrent papillary thyroid carcinoma and, like other cancers, are proposed to play a role in growth, metastasis and recurrence [25]. Anti-VEGF antibodies and other antiangiogenic therapies are currently in phase III clinical trials and a search is underway for good predictive markers of cancer response [68, 69].

Metastatic DTC cells express a somatostatin receptor subtype which can be tagged using a somatostatin analogue and detected via PET scanning. (68Ga) DOTATOC is one such somatostatin analogue. PET scanning with this molecule has not been shown to be more effective than (18F) FDG scanning. Higher affinity analogues exist but have not yet been studied in context of PET detection of recurrent thyroid cancer [36].

\section{Outcomes and Conclusions}

Well differentiated thyroid cancer recurs in $5-20 \%$ of patients. Cancer-related mortality has been estimated at $6.5 \%$ $[2,70]$. Survival of primary disease is arguably excellent with 10 year survival rates of $98 \%$ and $92 \%$ in papillary and follicular cancers respectively [71]. Forty-year survival rates are $94 \%$ and $84 \%$ [72].

Life expectancy for persistent disease is dependent on tumor characteristics, but has been shown to be only $60 \%$ that of the general population [3]. Roughly half of all patients with recurrent thyroid cancer will eventually die from disease [73]. Cancer-related mortality from multicentric recurrent papillary thyroid carcinoma was found to be $27.8 \%$ in one study [14]. An older study by Rossi et al puts forth treatment success rates at $73 \%$ for patients with nodal recurrence, $53 \%$ for local recurrences, and only $27 \%$ for distant recurrences [7]. Extrathyroidal extension may be a negative prognostic factor in survival of recurrent papillary cancer [10]. Thorough resection of primary disease decreases the risks of recurrence and secondarily decreases the risk of death from recurrent disease. Recurrence in the remnant thyroid or subcutaneous tissue has better outcomes than recurrence in the thyroid bed or surrounding tissue [74]. Primary disease staged T1-T3 has excellent 5, 10, and 15 year survival rates $(96 \%, 93 \%, 87 \%$ respectively). Both $\mathrm{T} 4$ and distant disease have increasingly worsened survivability [54]. Despite this handful of studies, there is no definitive answer yet on the long-term survivability of recurrent differentiated thyroid cancer.

\section{References}

1. http://www.cancer.gov/cancertopics/types/thyroid.

2. Todd WUt, Wenig BM. Thyroid follicular epithelial cellderived carcinomas: an overview of the pathology of primary and recurrent disease. Otolaryngol Clin North Am. 2008;41(6):1079-1094, vii-viii.

3. Cooper DS, Doherty GM, Haugen BR, Kloos RT, Lee SL, Mandel SJ, Mazzaferri EL, et al. Revised American Thyroid Association management guidelines for patients with thyroid nodules and differentiated thyroid cancer. Thyroid. 2009;19(11):1167-1214.

4. Shen WT, Ogawa L, Ruan D, Suh I, Kebebew E, Duh QY, Clark OH. Central neck lymph node dissection for papillary thyroid cancer: comparison of complication and recurrence rates in 295 initial dissections and reoperations. Arch Surg. 2010;145(3):272-275.

5. Sanders LE, Cady B. Differentiated thyroid cancer: reexamination of risk groups and outcome of treatment. Arch Surg. 1998;133(4):419-425.

6. Sanders LE, Silverman M. Follicular and Hurthle cell carcinoma: predicting outcome and directing therapy. Surgery. 1998;124(6):967-974.

7. Rossi RL, Cady B, Silverman ML, Wool MS, Horner TA. Current results of conservative surgery for differentiated thyroid carcinoma. World J Surg. 1986;10(4):612622.

8. Orlov S, Orlov D, Shaytzag M, Dowar M, Tabatabaie V, Dwek P, Yip J, et al. Influence of age and primary tumor size on the risk for residual/recurrent well-differentiated thyroid carcinoma. Head Neck. 2009;31(6):782-788.

9. Vaisman F, Shaha A, Fish S, Tuttle R. Initial therapy with either thyroid lobectomy or total thyroidectomy without radioactive iodine remnant ablation is associated with very low rates of structural disease recurrence in properly selected patients with differentiated thyroid cancer. Clin Endocrinol (Oxf). 2011.

10. Forest VI, Clark JR, Ebrahimi A, Cho EA, Sneddon L, Gao K, O'Brien C J. Central compartment dissection in thyroid papillary carcinoma. Ann Surg. 2011;253(1):123130.

11. Nascimento C, Borget I, Al Ghuzlan A, Deandreis D, Chami L, Travagli JP, Hartl D, et al. Persistent disease and recurrence in differentiated thyroid cancer patients with undetectable postoperative stimulated thyroglobulin level. Endocr Relat Cancer. 2011;18(2):R29-40.

12. Raef H, Alfadhli E, Al-Hajjaj A, Malabu UH, Al-Sobhi S, Rifai A, Al Nuaim A. High rate of persistent/recurrent disease among patients with differentiated thyroid cancer in Saudi Arabia: factors affecting nonremission. Ann 
Saudi Med. 2008;28(4):277-281.

13. Ort S, Goldenberg D. Management of regional metastases in well-differentiated thyroid cancer. Otolaryngol Clin North Am. 2008;41(6):1207-1218, xi.

14. Lin JD, Chao TC, Hsueh C, Kuo SF. High recurrent rate of multicentric papillary thyroid carcinoma. Ann Surg Oncol. 2009;16(9):2609-2616.

15. Tuttle RM, Leboeuf R. Follow up approaches in thyroid cancer: a risk adapted paradigm. Endocrinol Metab Clin North Am. 2008;37(2):419-435, ix-x.

16. Barollo S, Pennelli G, Vianello F, Watutantrige Fernando S, Negro I, Merante Boschin I, Pelizzo MR, et al. BRAF in primary and recurrent papillary thyroid cancers: the relationship with (131)I and 2-[(18)F]fluoro2-deoxy-D-glucose uptake ability. Eur J Endocrinol. 2010;163(4):659-663.

17. Yip L, Nikiforova MN, Carty SE, Yim JH, Stang MT, Tublin MJ, Lebeau SO, et al. Optimizing surgical treatment of papillary thyroid carcinoma associated with BRAF mutation. Surgery. 2009;146(6):1215-1223.

18. Cradic KW, Milosevic D, Rosenberg AM, Erickson LA, McIver B, Grebe SK. Mutant BRAF(T1799A) can be detected in the blood of papillary thyroid carcinoma patients and correlates with disease status. J Clin Endocrinol Metab. 2009;94(12):5001-5009.

19. Kebebew E, Weng J, Bauer J, Ranvier G, Clark OH, Duh QY, Shibru D, et al. The prevalence and prognostic value of BRAF mutation in thyroid cancer. Ann Surg. 2007;246(3):466-470; discussion 470-461.

20. Nikiforov YE. Molecular analysis of thyroid tumors. Mod Pathol. 2011;24 Suppl 2:S34-43.

21. Nikiforov YE. Molecular diagnostics of thyroid tumors. Arch Pathol Lab Med. 2011;135(5):569-577.

22. Mian C, Barollo S, Pennelli G, Pavan N, Rugge M, Pelizzo MR, Mazzarotto R, et al. Molecular characteristics in papillary thyroid cancers (PTCs) with no 131I uptake. Clin Endocrinol (Oxf). 2008;68(1):108-116.

23. Henderson YC, Shellenberger TD, Williams MD, ElNaggar AK, Fredrick MJ, Cieply KM, Clayman GL. High rate of BRAF and RET/PTC dual mutations associated with recurrent papillary thyroid carcinoma. Clin Cancer Res. 2009;15(2):485-491.

24. Howell GM, Carty SE, Armstrong MJ, Lebeau SO, Hodak SP, Coyne C, Stang MT, et al. Both BRAF V600E mutation and older age $(>/=65$ years) are associated with recurrent papillary thyroid cancer. Ann Surg Oncol. 2011;18(13):3566-3571.

25. Yu XM, Lo CY, Lam AK, Lang BH, Leung P, Luk JM. The potential clinical relevance of serum vascular endothelial growth factor (VEGF) and VEGF-C in recurrent papillary thyroid carcinoma. Surgery. 2008;144(6):934940; discussion 940-931.

26. Wang W, Wang $\mathrm{H}$, Teng $\mathrm{X}$, Mao C, Teng R, Zhao W, Cao J, et al. Clonal analysis of bilateral, recurrent, and metastatic papillary thyroid carcinomas. Hum Pathol. 2010;41(9):1299-1309.

27. Aygun N. Imaging of recurrent thyroid cancer. Otolaryngol Clin North Am. 2008;41(6):1095-1106, viii.

28. Park EK, Chung JK, Lim IH, Park do J, Lee DS, Lee MC, Cho BY. Recurrent/metastatic thyroid carcinomas false negative for serum thyroglobulin but positive by postherapy I-131 whole body scans. Eur J Nucl Med Mol Imaging. 2009;36(2):172-179.

29. Yim JH, Kim WB, Kim EY, Kim WG, Kim TY, Ryu JS, Gong G, et al. The outcomes of first reoperation for locoregionally recurrent/persistent papillary thyroid carcinoma in patients who initially underwent total thyroidectomy and remnant ablation. J Clin Endocrinol Metab. 2011;96(7):2049-2056.

30. Kloos RT. Thyroid cancer recurrence in patients clinically free of disease with undetectable or very low serum thyroglobulin values. J Clin Endocrinol Metab. 2010;95(12):5241-5248.

31. Aras G, Gultekin SS, Kucuk NO. The additive clinical value of combined thyroglobulin and antithyroglobulin antibody measurements to define persistent and recurrent disease in patients with differentiated thyroid cancer. Nucl Med Commun. 2008;29(10):880-884.

32. Shin JJ, Milas M. Detection of disease recurrence in differentiated thyroid cancer. Minerva Chir. 2010;65(1):101-116.

33. Kim JH, Kim MS, Yoo SY, Lim SM, Lee GH, Yi KH. Stereotactic body radiotherapy for refractory cervical lymph node recurrence of nonanaplastic thyroid cancer. Otolaryngol Head Neck Surg. 2010;142(3):338-343.

34. Johnson NA, LeBeau SO, Tublin ME. Imaging surveillance of differentiated thyroid cancer. Radiol Clin North Am. 2011;49(3):473-487, vi.

35. Choi JW, Lee JH, Baek JH, Choi BS, Jeong KS, Ryu JS, Kim TY, et al. Diagnostic accuracy of ultrasound and 18-F-FDG PET or PET/CT for patients with suspected recurrent papillary thyroid carcinoma. Ultrasound Med Biol. 2010;36(10):1608-1615.

36. Middendorp M, Selkinski I, Happel C, Kranert WT, Grunwald F. Comparison of positron emission tomography with [(18)F]FDG and [(68)Ga]DOTATOC in recurrent differentiated thyroid cancer: preliminary data. Q J Nucl Med Mol Imaging. 2010;54(1):76-83.

37. Sanz Viedma S, Borrego Dorado I, Rodriguez Rodriguez JR, Navarro Gonzalez E, Vazquez Albertino R, Fernandez Lopez R, Agudo Martinez A. [Use of 18F FDG-PET in patients with suspicion of recurrent differentiated thyroid cancer by elevated antithyroglobulin antibodies levels and negative (1)(3)(1)I scan]. Rev Esp Med Nucl. 2011;30(2):77-82.

38. Razfar A, Branstetter BFt, Christopoulos A, Lebeau SO, Hodak SP, Heron DE, Escott EJ, et al. Clinical usefulness of positron emission tomography-computed tomog- 
raphy in recurrent thyroid carcinoma. Arch Otolaryngol Head Neck Surg. 2010;136(2):120-125.

39. Lal G, Fairchild T, Howe JR, Weigel RJ, Sugg SL, Menda Y. PET-CT scans in recurrent or persistent differentiated thyroid cancer: is there added utility beyond conventional imaging? Surgery. 2010;148(6):1082-1089; discussion 1089-1090.

40. Miller ME, Chen Q, Elashoff D, Abemayor E, St John M. Positron emission tomography and positron emission tomography-CT evaluation for recurrent papillary thyroid carcinoma: meta-analysis and literature review. Head Neck. 2011;33(4):562-565.

41. Grant CS, Thompson GB, Farley DR, Richards ML, Mullan BP, Hay ID. The value of positron emission tomography in the surgical management of recurrent papillary thyroid carcinoma. World J Surg. 2008;32(5):708-715.

42. Davison JM, Stocker DJ, Montilla-Soler JL, Jurgens JS, Allen TW, Holley TS, Stack AL. The added benefit of a dedicated neck F-18 FDG PET-CT imaging protocol in patients with suspected recurrent differentiated thyroid carcinoma. Clin Nucl Med. 2008;33(7):464-468.

43. Lee DH, Kang WJ, Seo HS, Kim E, Kim JH, Son KR, $\mathrm{Na}$ DG. Detection of metastatic cervical lymph nodes in recurrent papillary thyroid carcinoma: computed tomography versus positron emission tomography-computed tomography. J Comput Assist Tomogr. 2009;33(5):805810 .

44. Ahmadzadehfar H, Biermann K, Sabet A, Biersack HJ. A letter regarding diagnostic accuracy of ultrasound and 18-F-FDG PET or PET/CT for patients with suspected recurrent papillary thyroid carcinoma. Ultrasound Med Biol. 2011;37(5):840; author reply 841.

45. Kim DW, Choo HJ, Park JS, Lee EJ, Kim SH, Jung SJ, Ryu JH. Ultrasonography-guided fine-needle aspiration cytology for thyroid nodules: An emphasis on one-sampling and biopsy techniques. Diagn Cytopathol. 2011.

46. Frates MC. Ultrasound in recurrent thyroid disease. Otolaryngol Clin North Am. 2008;41(6):1107-1116, viii.

47. Hahn SY, Shin JH, Han BK, Ko EY, Kang SS, Chung $\mathrm{JH}, \mathrm{Kim} \mathrm{JH}$, et al. Predictive factors related to the recurrence at US-guided fine needle aspiration in postoperative patients with differentiated thyroid cancer. Clin Endocrinol (Oxf). 2011;74(2):270-275.

48. Berger F, Friedrich U, Knesewitsch P, Hahn K. Diagnostic 131I whole-body scintigraphy 1 year after thyroablative therapy in patients with differentiated thyroid cancer: correlation of results to the individual risk profile and long-term follow-up. Eur J Nucl Med Mol Imaging. 2011;38(3):451-458.

49. Ondik MP, Dezfoli S, Lipinski L, Ruggiero F, Goldenberg D. Secondary central compartment surgery for thyroid cancer. Laryngoscope. 2009;119(10):1947-1950.

50. Hughes DT, Doherty GM. Central neck dissection for papillary thyroid cancer. Cancer Control. 2011;18(2):83-
88.

51. Kim MK, Mandel SH, Baloch Z, Livolsi VA, Langer JE, Didonato L, Fish S, et al. Morbidity following central compartment reoperation for recurrent or persistent thyroid cancer. Arch Otolaryngol Head Neck Surg. 2004;130(10):1214-1216.

52. Johnson S, Goldenberg D. Intraoperative monitoring of the recurrent laryngeal nerve during revision thyroid surgery. Otolaryngol Clin North Am. 2008;41(6):11471154, ix.

53. Pai SI, Tufano RP. Reoperation for recurrent/persistent well-differentiated thyroid cancer. Otolaryngol Clin North Am. 2010;43(2):353-363, ix.

54. Hessel A, Chalian AA, Clayman GL. Surgical management of recurrent thyroid cancer. Neuroimaging Clin $\mathrm{N}$ Am. 2008;18(3):517-525, viii.

55. Clayman GL, Shellenberger TD, Ginsberg LE, Edeiken BS, El-Naggar AK, Sellin RV, Waguespack SG, et al. Approach and safety of comprehensive central compartment dissection in patients with recurrent papillary thyroid carcinoma. Head Neck. 2009;31(9):1152-1163.

56. Roh JL, Kim JM, Park CI. Central compartment reoperation for recurrent/persistent differentiated thyroid cancer: patterns of recurrence, morbidity, and prediction of postoperative hypocalcemia. Ann Surg Oncol. 2011;18(5):1312-1318.

57. Ondik MP, McGinn J, Ruggiero F, Goldenberg D. Unintentional parathyroidectomy and hypoparathyroidism in secondary central compartment surgery for thyroid cancer. Head Neck. 2010;32(4):462-466.

58. Roh JL, Park JY, Rha KS, Park CI. Is central neck dissection necessary for the treatment of lateral cervical nodal recurrence of papillary thyroid carcinoma? Head Neck. 2007;29(10):901-906.

59. Cheah WK, Arici C, Ituarte PH, Siperstein AE, Duh QY, Clark OH. Complications of neck dissection for thyroid cancer. World J Surg. 2002;26(8):1013-1016.

60. Schuff KG, Weber SM, Givi B, Samuels MH, Andersen PE, Cohen JI. Efficacy of nodal dissection for treatment of persistent/recurrent papillary thyroid cancer. Laryngoscope. 2008;118(5):768-775.

61. Udelsman R. Treatment of persistent or recurrent papillary carcinoma of the thyroid--the good, the bad, and the unknown. J Clin Endocrinol Metab. 2010;95(5):20612063.

62. Ondik MP, Tulchinsky M, Goldenberg D. Radioguided reoperative thyroid and parathyroid surgery. Otolaryngol Clin North Am. 2008;41(6):1185-1198, x.

63. Sippel RS, Elaraj DM, Poder L, Duh QY, Kebebew E, Clark OH. Localization of recurrent thyroid cancer using intraoperative ultrasound-guided dye injection. World J Surg. 2009;33(3):434-439.

64. Shaha AR. Localization of recurrent thyroid cancer: a technical challenge. Ann Otol Rhinol Laryngol. 
2009;118(7):479-480.

65. McCoy KL, Yim JH, Tublin ME, Burmeister LA, Ogilvie JB, Carty SE. Same-day ultrasound guidance in reoperation for locally recurrent papillary thyroid cancer. Surgery. 2007;142(6):965-972.

66. Yoshio K, Sato S, Okumura Y, Katsui K, Takemoto M, Suzuki E, Katayama N, et al. The local efficacy of I-131 for F-18 FDG PET positive lesions in patients with recurrent or metastatic thyroid carcinomas. Clin Nucl Med. 2011;36(2):113-117.

67. Jun JY, Manni A. Medical management of persistent or recurrent differentiated thyroid carcinoma. Otolaryngol Clin North Am. 2008;41(6):1241-1260, xi-xii.

68. Lalami Y, Awada A. Recurrent thyroid cancer: a molecular-based therapeutic breakthrough. Curr Opin Oncol. 2011;23(3):235-240.

69. Rovere RK, Awada A. Treatment of recurrent thyroid cancers--is there a light in the horizon? Curr Opin Oncol. 2008;20(3):245-248.
70. Ruggiero FP, Fedok FG. Outcomes in reoperative thyroid cancer. Otolaryngol Clin North Am. 2008;41(6):12611268, xii.

71. Gilliland FD, Hunt WC, Morris DM, Key CR. Prognostic factors for thyroid carcinoma. A population-based study of 15,698 cases from the Surveillance, Epidemiology and End Results (SEER) program 1973-1991. Cancer. 1997;79(3):564-573.

72. Mazzaferri EL, Kloos RT. Clinical review 128: Current approaches to primary therapy for papillary and follicular thyroid cancer. J Clin Endocrinol Metab. 2001;86(4):1447-1463.

73. Coburn M, Teates D, Wanebo HJ. Recurrent thyroid cancer. Role of surgery versus radioactive iodine (I131). Ann Surg. 1994;219(6):587-593; discussion 593-585.

74. Ito Y, Higashiyama T, Takamura Y, Kobayashi K, Miya A, Miyauchi A. Prognosis of patients with papillary thyroid carcinoma showing postoperative recurrence to the central neck. World J Surg. 2011;35(4):767-772. 